\title{
CONTRO LE BORIE “RITORNANTI”: PER UN SANO USO DELLA CRITICA
}

\author{
Giuseppe Cacciatorel
}

\begin{abstract}
RIASSUNTO: L'Autore ritiene che la critica vichiana delle borie non si riferisca solo a nazioni, culture e teorie filosofico-teologiche concentrate a discutere sull'origine oscura e favolosa dei tempi e sulla relazione tra storia sacra e storie profane. Ma è anche un dispositivo etico-filosofico che invita l'umanità, ormai al culmine del processo di incivilimento, a guardarsi da ogni forma di boria delle nazioni (in termini contemporanei: superbia etnocentrica), così come da ogni manifestazione boriosa da parte dei dotti (ovvero da ogni forma di filosofia e di teoria astrattamente antropocentrica).
\end{abstract}

PAROLE CHIAVE: Etnocentrismo Antropocentrismo. Etica. Interculturalità. Giambattista Vico.

1. In un saggio recente (CACCIATORE, 2011) pubblicato nella Rivista di Filosofia di Torino, ho affrontato il tema delle borie in Vico, considerato principalmente in relazione al significato che esse assumono nell'articolato e complesso rapporto che in Vico si istaura tra etica e filosofia della storia. In quelle pagine, come in queste che ora sto esponendo, ho dato maggiore spazio al problema filosofico ed etico-politico rappresentato dalle borie, piuttosto che dalle questioni, pur importanti, connesse all'indagine filologica e storico-esegetica, così come essa si profila in ordine al giudizio vichiano sulle remote antichità di popoli e di idee all'origine del processo di incivilimento del genere umano.

Il rapporto che già nel titolo ho creduto di poter istituire tra borie e critica, si fonda su due precisi convincimenti: il primo riguarda l'uso che

\footnotetext{
${ }^{1}$ Professore ordinario di Storia della filosofia presso il Dipartimento di Studi umanistici dell'Università degli studi di Napoli Federico II. È Accademico dei Lincei e membro di numerose e prestigiose Accademie, fra cui l'Accademia Pontaniana e la Società Nazionale di Scienze, Lettere e Arti di Napoli. Inoltre è membro del Consiglio scientifico della Graduierten Schule "Friedrich Schlegel" della Freie Universität di Berlino. Esponente dello storicismo critico-problematico di scuola napoletana, è studioso noto anche all'estero, con una vastissima produzione scientifica che annovera numerosi saggi e volumi su grandi figure della filosofia tedesca (Dilthey, Bloch, Cassirer), italiana (Vico, Croce, Labriola, Gramsci), ispanica (Ortega y Gasset, Zambrano) e ispano-americana (Nicol, Zea). Da diversi anni - con allievi e studiosi più giovani - sta conducendo ricerche sulla filosofia dell'interculturalità e sulle questioni etico-politiche connesse al rapporto fra le culture. Sue pubblicazioni più recenti sono: Sulla filosofia spagnola. Saggi e ricerche (il Mulino, 2013) e Problemi di filosofia della storia nell'età di Kant e di Hegel. Filologia, critica, storia civile (Aracne, 2013).
} 
Vico fa del concetto e della storia stessa delle borie e che io definirei, con consapevole forzatura ermeneutica, come processo di deideologizzazione di ogni idea di primato di popoli, di culture e di filosofie; il secondo deriva dalla centralità che nel pensiero vichiano assume l'idea di critica, l'alterità dialettica insopprimibile, come ben sanno gli studiosi di Vico, dell'idea di topica.

2. È ben noto il legame che Vico istituisce tra metafisica e storia, tra ordine delle idee e storicità del mondo umano. Ma quella di Vico è anche una metafisica della mente - quella figura che è al centro dell'universo immaginativo e a un tempo epistemologico di Vico -, la cui funzione si svolge e si esalta come continua ricerca delle possibilità e dei luoghi in cui si costituisce e si manifesta la relazione tra la finitezza del fatto umano e l'infinità del vero divino. La storia, che è un aspetto della finitezza dell'uomo, si costituisce in questo spazio di differenza tra il fare divino e il fare umano. Tutto ciò che caratterizza l'umano ha origine, per così dire, dal difetto originario della sua mente, dall'impossibilità di poter contenere in sé l'infinità delle cose. Soltanto grazie all'astrazione, da un lato, e ai processi conoscitivi della scienza storica e della filologia, dall'altro, l'uomo può riconoscere e ricostruire gli elementi delle cose e organizzarli in immagini, simboli e idee (cf. CACCIATORE, 2009). Proprio la consapevolezza dei limiti della mente umana conferisce, come vedremo, al tema vichiano delle borie una connotazione che non è legata solo agli inizi oscuri ed incerti del mondo umano (ed alla pretesa di nazioni e di dotti di costituire per sé una immagine di esclusiva ed unica sapienza delle origini), ma è relativa ad una idea della natura umana perfettibile e spesso fuorviata da errori e da presunzioni. La metafisica, nel senso appunto di una "metafisica della mente umana", diventa, come è stato opportunatamente osservato (OTTO, 1992), il motivo fondamentale dell'opera vichiana, già delineato nel Liber metaphysicus e, poi, definitivamente elaborato nella Scienza nuova. E si tratta di una metafisica del tutto particolare, che non rientra ormai più nelle classiche tipologie che più tardi Kant avrebbe sottoposto a critica demolitrice, giacché essa è, fin dall'inizio, una "metafisica della mente umana storica". Il punto di vista cartesiano è totalmente rovesciato, giacché il solo criterio di evidenza della verità, basato sulla coscienza del cogito, non è capace di fondare la scienza umana che, proprio per questo, ha bisogno di radicarsi in una metafisica della mente nella quale si costruisce la necessaria interazione di vero e fatto, razionalità e ingegno, critica e topica (cf. CACCIATORE, 2002). La metafisica vichiana della storia, come si vedrà meglio anche più innanzi, 
postula consapevolmente il grande problema filosofico del rapporto tra fatti e idee, temporalità ed eternità. La storia ideale eterna, se è da intendere come storia delle idee, lo è non certo nel senso della riduzione della storia a idee metafisiche, predefinite e preesistenti alla coscienza dell'uomo, ma in quello di idee costruite dalla mente umana, la cui capacità creativa muove lo stesso agire sociale e storico dell'uomo.

È sulla base di questo forte background teoretico che bisogna, a mio parere, rileggere e a ripensare quelle degnità della Scienza nuova del 1744 nelle quali Vico parla di due "spezie di borie": quella delle nazioni e quella dei dotti. Si trattava, peraltro, di una ben evidente conseguenza di una precisa premessa di ordine, a un tempo, epistemologica e valoriale (nel senso di una opzione di tipo etico). Vico, infatti, aveva introdotto la serie delle degnità con la ben nota affermazione secondo la quale l'uomo, proprio a causa della indeterminatezza della sua mente, quando questa "[...] si rovesci nell'ignoranza", tende a fare di sé stesso la regola dell'universo (VICO, 1744, t. I, capov. 120, p. 494.). Insomma, come più avanti Vico osserva, citando una massima di Tacito, omne ignotum pro magnifico est. Come molti studiosi di Vico hanno giustamente sostenuto, la fonte delle riflessioni vichiane è qui individuabile in Bacone e nella sua critica degli idola tribus e degli idola specus. Anche Vico è alla ricerca delle ragioni che hanno causato la maggior parte degli errori commessi dai dotti, come dalle nazioni, riguardo ai "principi dell'umanità". Ma la fonte principale dell'errore è da individuare ancora una volta nel peculiare modo d'essere della mente umana, "[...] ch'ove gli uomini delle cose lontane e non conosciute non possono fare alcuna idea, le stimano dalle cose loro conosciute e presenti" (VICO, 1744, capov. 122, p. 494-495). Non solo ma proprio l'ignoranza delle origini dell'umanità spinge le borie dei dotti e delle nazioni a ritenerle rozze ed oscure.

Come ho detto innanzi, del discorso vichiano sulle borie a me non interessa tanto né la discutibile ed errata scansione cronologica adottata da Vico, né i modi e i contenuti della sua ricostruzione della storia dei popoli favolosi ed antichi. Il tema delle "borie" mette in campo un discorso che riguarda, da un lato, il corso delle nazioni dal punto di vista della filosofia della storia e, dall'altro, una valutazione etica - non arrischio l'impropria espressione di giudizio morale - sull'eccesso di borie nella storia delle relazioni tra popoli e civiltà. Il "fonte inesausto di tutti gli errori" in cui incorsero intere nazioni e falangi di dotti, riguarda i principi stessi dell'umanità, la cui presunta oscurità e rozzezza, avvertite dalle comunità e ragionate dai dotti, 
“[...] autorizzano gli antichi popoli a pensare di essere stati prima degli altri capaci di ritrovare i comodi della vita umana e conservare le memorie delle loro cose fin dal principio del mondo" (VICO, 1744, capov. 125, p. 495). I verbi adoperati da Vico non sono scelti a caso, ma, come osserva Battistini - con la cui interpretazione io convengo -, testimoniano di come il filosofo napoletano sapesse operare una distinzione che chiarisce, anche sotto l'aspetto epistemologico, il motivo dell'articolazione delle due borie. Una cosa, dunque, è l'immediato avvertire, la "[...] percezione intuitiva di una collettività (nazioni)", altra cosa, invece, è il meditato ragionare, la "[...] razionalità riflessa degli studiosi (dotti)” (BATTISTINI, 1990, p. 1519).

Come è noto, il discorso vichiano sulle borie poggia fondamentalmente su una ricostruzione filologica e storico-erudita (penso al tema, per fare un solo esempio, della storia sacra o a quello della cronologia biblica), talvolta discutibile e persino errata. Resta però l'importanza e la centralità che Vico assegna allo strumento della ricerca storica e filologica che, in questo caso, si manifesta come rispetto non borioso e non presuntuoso della critica storico-filologica. La stessa progressiva dissoluzione delle borie è resa possibile proprio dal manifestarsi di quella verità che non può essere in nessun modo posta in dubbio: "[...] che questo mondo civile egli certamente è stato fatto dagli uomini". Nel capoverso precedente Vico aveva dipinto a tinte fosche la conseguenza delle borie.

Laonde, perché la boria delle nazioni, d'essere stata ogniuna la prima del mondo, ci disanima di ritruovare i princìpi di questa Scienza da' filologi; altronde la boria de' dotti, i quali vogliono ciò ch'essi sanno essere stato eminentemente inteso fin dal principio del mondo, ci dispera di ritruovargli da' filosofi: quindi, per questa ricerca, si dee far conto come se non vi fussero libri nel mondo.

È, come sanno i lettori di Vico, proprio a questo punto che, con immagine tradizionalmente retorica e letteraria, appare questo lume eterno che squarcia "[...] la densa notte di tenebre ond'è coverta la prima da noi lontanissima antichità": il mondo storico, il mondo delle nazioni, "[...] del quale, perché l'avevano fatto gli uomini, ne potevano conseguire la scienza gli uomini" (VICO, 1744, capov. 330 e 331, p. 541-542).

Insomma, ammessi e riconosciuti tutti i limiti che mostrava Vico rispetto alla cultura filosofica e scientifica dei suoi contemporanei e tutto il peso del tradizionalismo cattolico che gravava sul suo pensiero, che cos'è la 
Scienza nuova se non una delle opere epochemachende, per usare il termine tedesco, e che cos'è il pensiero di Vico se non una indagine storica e filosofica radicalmente nuova sull'origine delle cose, una ricerca di "pruove" filosofiche e filologiche pur dentro la cornice ortodossa della religione? Mi pare che a testimonianza di ciò possa bastare anche la sola indicazione del preciso obiettivo che egli ha individuato per la nuova scienza (Nova scientia tentatur) di ricostruire, a partire dalle origini, così delle cose, come del mondo civile, la storia e il modo d'essere delle nazioni, lungo un percorso che certamente è illuminato dalla storia ideale eterna ma che è anche e soprattutto prodotto dell'agire storico dell'uomo e delle istituzioni in cui si manifesta. D'altronde è con l'apparire degli elementi fondativi dell'umanità, la religione innanzitutto e il formarsi dei nuclei primitivi di aggregazione comunitaria, che le storie profane delle nazioni nel loro originarsi diventano il vero luogo di sperimentazione e certificazione non solo delle scienze storico-filologiche, ma anche degli stessi principi di filosofia della storia.

I testi vichiani, come quelli di tutti i grandi filosofi, sono naturalmente soggetti alla pluralità e diversità delle interpretazioni, talvolta anche radicalmente confliggenti. Quella sulla quale ho qui argomentato per segnalare, a proposito delle borie, non solo la loro interpretazione in chiave di filosofia universalistica della storia, ma anche la loro curvatura etica ed antropologica, è una delle possibili chiavi di lettura, esposte, come è giusto che sia, alle critiche, alle correzioni e, spero, anche alle convergenze. Credo che si possa sostenere che la critica vichiana delle borie non riguarda solo nazioni e culture e teorie filosofico-teologiche concentrate a discettare sull'origine oscura e favolosa dei tempi e sulla relazione tra storia sacra e storie profane. Si tratta anche di un dispositivo etico-filosofico che invita l'umanità, ormai al culmine del processo di incivilimento, a guardarsi da ogni forma di boria delle nazioni (potremmo dire in termini contemporanei, da ogni forma di superbia etnocentrica), così come da ogni manifestazione boriosa da parte dei dotti (potremmo dire, da ogni forma di filosofia e di teoria astrattamente antropocentrica) (cf. DIANA, 2011; PONS, 1997).

Resta da chiedersi se abbia plausibilità storico-testuale e interpretativa quel collegamento che, sin dal titolo di questo mio intervento, ho individuato tra la critica a un momento particolare della vita storica dell'umanità, quello delle borie delle antiche nazioni e degli antichi dotti, e un momento, per così dire filosofico e universale costituito dall'arte critica vichiana, sulla quale, 
già nella "spiegazione della dipintura" (VICO, 1744, capov. 7, p. 419), Vico basava la necessaria interazione tra filosofia e filologia.

Il fatto che Vico parli esplicitamente di "nuova arte critica" testimonia della piena consapevolezza che egli ha nel ritenere radicalmente innovativa la sua proposta, al contempo metodologica e filosofico-sistematica, rispetto alle altre "critiche", tanto quella meramente filologico-erudita, quanto quella razionalistico-metafisica (cf. VERENE, 1981; CAPONIGRI, 1982-1983). In una importante lettera del 1729 a Francesco Saverio Estevan, Vico riprendendo un concetto che era stato al centro del De Ratione - esalta la virtù dell'ingegno, che è "l'unico padre di tutte le invenzioni". L'esercizio della Topica (erroneamente trascurato e persino disprezzato dai "loici" contemporanei), proprio perché mette in moto un percepire che viene prima di ogni riflessione concettuale, è in grado di allestire, per il giudizio stesso, una critica,

[...] quanto più accertata, tanto più utile alla Scienza per le Sperienze in Natura, e per gli nuovi ritrovati dell'Arti; Utile alla Prudenza per ben fermare le congetture delle cose o fatte per giustamente giudicarle, o' da farsi per utilmente condurle. (VICO, 1992, p. 143-144).

La critica alla quale, dunque, qui Vico pensa non ha nulla in comune né con quella "metafisica", che finisce per smarrirsi nello scetticismo e per attutire la originaria tendenza della mente umana al senso comune (e, cosa ancora più grave, ricaccia l'uomo dalla comunanza civile alla solitudine egoistica delle proprie utilità), né con quella "erudita", che "[...] di nulla serve a far sappienti coloro, che la coltivano" (VICO, 1992, p. 144). La nuova arte critica, come ben si può vedere, non ha soltanto una valenza metodologica e gnoseologica. $\mathrm{Al}$ di là di questa traspare con nettezza il fondamentale orientamento "pratico" della filosofia vichiana. Se l'arte critica è in grado di giudicare come l'operare dell'uomo debba conformarsi alle circostanze in cui si trova collocato, allora essa è " $[. .$.$] critica sappientissima dell'arbitrio umano, il qual'è per sua natura$ incertissimo e perciò sommamente necessaria agli huomini di Stato" (VICO, 1992, p. 145). Per questo, Vico disapprova esplicitamente le tendenze, che egli addebita all'invadenza del metodo cartesiano, di quella critica che vorrebbe trasportare i metodi dalle matematiche alle altre scienze che, riducendo tutto alla presunta chiarezza dell'intelletto, fiacca gli ingegni e li priva di ogni vigore.

Il circolo filosofia-storia-filologia - fondato sistematicamente sul rapporto originario di conversione tra verum e factum e sulla relazione metodica tra critica 
e topica - si salda nell'unitarietà della nuova scienza, nei rimandi necessari tra il suo essere studio delle forme espressive dell'esperienza umana (la poesia, la storia, il diritto, la lingua) e il suo costituirsi come filosofia dell'autorità, la quale, se, da un lato, è tesa a meditare una "[...] metafisica innalzata a contemplare la mente del Gener'Umano, e quindi Iddio per l'attributo della Provvedenza", dall'altro, ha bisogno di una critica che accerti ciò che storia e poesia hanno detto e scritto sull'origine delle nazioni (VICO, 1992, p. 145).

La scienza vichiana è nuova (e, aggiungeremmo, consapevolmente moderna), sa di essere nuova, perché l'“arte critica" che vuole fondare è innanzitutto rivolta alla "ricerca del vero sopra gli autori delle nazioni" e sa, inoltre, di essere nuova perché per la prima volta la "filosofia si pone ad esaminare la filologia" (cioè, come spiega Vico, tutto ciò che dipende dall'arbitrio umano, le storie delle lingue, dei costumi, delle vicende politiche dei popoli).

In molte mie pagine teoretiche su Vico (mai disgiunte dalla lettura e dalla interpretazione dei testi) ho sostenuto che si poteva ritrovare in Vico se non una chiarificazione definitiva, certamente qualche elemento di riflessione su una delle questioni cruciali della nostra contemporaneità e cioè se non sia possibile riproporre un universalismo etico che sappia coniugare la normatività del principio e la differenza storico-culturale, la necessità di costruire schemi, modelli e paradigmi e ciò che l'esperienza, attraverso la ricerca storico-analogica, offre al nostro sguardo e alla nostra comprensione. Ed è infine ancora da ben precise intuizioni filosofiche vichiane che può discendere un'idea di critica non soltanto epistemica e trascendentale, ma anche e soprattutto etica, quando essa si fa critica alla sempre possibile deriva dogmatica dell'universalismo: la necessità, cioè, che la dimensione generale non si separi mai dalla storicità determinata delle nazioni civili e dall'ineliminabile patrimonio delle differenze storico-culturali delle singole comunità. Ciò non contraddice, nell'economia del discorso vichiano, l'esistenza di un modello di filosofia della storia metafisico-teologica, il che, tra l'altro, contribuisce a spiegare l'insistenza vichiana a ritrovare quasi esclusivamente le "tracce" e le "rovine" del passato. E, tuttavia, tutto questo non mette in discussione la novità e l'originalità del metodo della scienza nuova, di un paradigma critico e "scientifico" consapevolmente rivolto a determinare e a descrivere le strutture storico-antropologiche, le istituzioni civili e giuridiche le stesse articolazioni sociali sia pur considerate nella loro arcaicità. Universalità dei principi e differenza, filosofia della storia e scienza empirica di essa che è in grado di trovare (l'originario invenire degli antichi dotti non boriosi) le distinzioni e le 
determinazioni "tra tutti i possibili umani", in modo tale da poter risalire agli “ $[. .$.$] incominciamenti più semplici e più naturali” di " [. .$.$] tante, sì varie \mathrm{e}$ diverse cose" (VICO, 1744, capov. 630, p. 729). E non mette in discussione neanche altri due essenziali contributi vichiani alla riflessione filosofica moderna e contemporanea. Infatti è dall'ordine metafisico dell'universo che discende la regola - che diventa nel corso del processo di incivilimento di popoli e culture tutta umana e mondana - della "sapienza volgare" (VICO, 1725 , v. 2 , p. 1008 ss.), cioè quella che dà vita al "senso comune", che è ritrovabile in ogni popolo e che è alla base della " [...] nostra vita socievole in tutte le nostre umane azioni” (VICO, 1725, p. 1009) ${ }^{2}$.

Lo strumento metodico della critica, quello strumento che aveva consentito a Vico di prendere le distanze dagli effetti negativi delle borie sulla considerazione storica della genesi delle antiche nazioni e sull'eccesso di dogmatismo ovvero di relativismo delle filosofie dei dotti sapienti delle origini, non perde, neanche oggi, di efficacia e di validità contro le tante borie ritornanti dell'epoca contemporanea. Sarebbe naturalmente fin troppo facile, e probabilmente scorretto da un punto di vista strettamente filologico ed ermeneutico, esercitarsi, anche riguardo al concetto di critica, in una operazione di precorrimento: di un Vico cioè anticipatore della rivoluzione criticistica kantiana e di tutto ciò che da questa rivoluzione si riverberò nel campo non soltanto della filosofia e della scienza. E, tuttavia, Vico resta tra i maggiori protagonisti di una "rivoluzione" concettuale e, a un tempo, ideologica, che mette al centro un'idea moderna di critica che oltrepassa gli ultimi residui metafisici e teologici e diviene - come osserva Koselleck - il luogo di maggiore visibilità della separazione tra ragione e rivelazione (KOSELLECK, 1972, p. 122-123). Anche se il Vico citato dallo storico tedesco è quello del De ratione - e dunque ancora dentro il modello razionalistico cartesiano -, tuttavia egli già ne coglie le prime decisive movenze verso una critica, che avrà il suo apogeo con Kant, che mette in discussione le rigidità e i dommatismi della ragione medesima. Ciò che ora si profila è un'idea di ragione come processo critico, sempre aperto e rinnovantesi, di accertamento della verità. Come ben sosteneva Cassirer,

[...] tutto il secolo XVIII intende la ragione in questo significato. Esso non la considera come un fisso contenuto di cognizioni, di principi, di

\footnotetext{
${ }^{2}$ Naturalmente è da ricordare la famosa definizione che del senso comune Vico dà nell'ultima edizione della sua opera. "Il senso comune è un giudizio senza alcuna riflessione, comunemente sentito da tutto un ordine, da tutto un popolo, da tutta una nazione o da tutto il gener'umano" (VICO, 1744, capov. 142, p. 498-499).
} 
verità, quanto piuttosto come una facoltà, come una forza che si può comprendere pienamente soltanto nel suo esercizio e nella sua esplicazione. (CASSIRER, 1970, p. 31).

In questo movimento di trasfigurazione dell'idea di critica, Vico porta un'originale idea di ragione. Il filosofo napoletano rappresenta l'“altra faccia" della modernità: quella che intenzionalmente ha utilizzato la fantasia e il fare poetico come critica ad ogni eccesso di logocentrismo. Cartesio resta, per il filosofo napoletano, punto obbligato di riferimento per chi voglia capire quanta importanza abbiano avuto per il pensiero moderno la scoperta della centralità del soggetto e il metodo di ricerca e verifica della verità. Ma ciò non gli ha impedito, fin dai primi scritti, di criticarne il riduzionismo logicorazionale. Ciò che mi è parso importante sottolineare è che tale critica non è meramente tradizionalistica, né soltanto preoccupata di recuperare la tradizione umanistica. Vico è alla ricerca di una nuova, più ampia, razionalità, capace di ritrovare topicamente i luoghi della fantasia, della corporeità, delle emozioni. Per questo credo si possa sostenere che, come la critica della ragione, anche la critica poetico-fantastica, possa indicare le condizioni di possibilità dell'agire umano. È in questo senso, allora, che si può chiarire il mio convincimento che Vico rappresenti l'altra faccia della modernità, quella della capacità inventiva e immaginativa, del verosimile e dei suoi eventi. Non so e non mi interessa sapere se qualcuno l'ha detto prima di me, ma si potrebbe dire della Scienza nuova, che è una vera e propria critica della ragione poetica (cf. CACCIATORE, 2012).

Ho detto prima che diffido dai facili e generalizzanti schemi del precursorismo, ma non riesco a sottrarmi al convincimento che questa critica della ragione poetica possa confrontarsi e affiancarsi alla diltheyana critica della ragione storica e all'orteghiano raziovitalismo. La critica della ragione di Dilthey è volta a cogliere, da un lato, i contenuti delle scienze dello spirito e, dall'altro, a studiarne le forme della loro esistenza storica nella successione degli Erlebnisse. La nuova critica storica della ragione diviene allora il fondamento teoretico che ci aiuta a comprendere "come la situazione di coscienza e l'orizzonte di un'età costituiscano sempre il presupposto per la maniera determinata in cui essa considera il mondo storico". Storia e autoriflessione logica e gnoseologica (topica e critica aveva detto Vico) ci aiutano a capire i modi in cui " [....] dall' Erleben di ciò che è accaduto sorge la connessione intuitiva e conoscitiva del mondo storico-sociale dell'uomo” (DILTHEY, 1954, p. 48). Anche il modello di ragione orteghiana non respinge il procedimento logico e 
conoscitivo dell'analisi e della scomposizione dei dati reali, ma questa ragione si presenta anche come "una breve zona di chiarezza che si apre dentro strati insondabili di irrazionalità". Per questo non è sufficiente il carattere formale e operativo, e dunque la ragione viene costantemente a intrecciarsi con un metodo intuitivo. "Ragionare - scrive Ortega - è un puro combinare visioni irragionevoli” (ORTEGA Y GASSET, 2005, p. 721-722).

È un filo conduttore quello che sto qui proponendo e non una ricerca compiuta e meglio organizzata nelle sue parti che ho in animo di poter avviare e concludere in un futuro spero non remoto. Per questo continuo a proporre suggestioni ed intuizioni più che argomenti e interpretazioni come quella con la quale concludo e che, guardando ancora una volta a quell'idea di una vichiana critica della ragione poetica, si affida a una pensosa e convincente riflessione di Enzo Paci.

La sapienza poetica è una topica sensibile dei primi autori della humanitas [...]. La topica è la tipica, il genere poetico, l'idea visiva concreta, l'eidetica, l'operazione precategoriale, la scienza della Lebenswelt, la fenomenologia. La critica è il giudicare le cose [...]. Il giudicare critico, distaccato dalla topica, è il giudizio non fondato, la logica separata dalla sua fondazione, la logica degli addottrinati. (PACI, 1973, p. 56ss.).

La logica, aggiungeremmo noi, dei boriosi non solo della filosofia e della politica, della storia e della cultura, ma anche della vita e delle sue relazioni interindividuali e interculturali.

CACCIATORE, Giuseppe. Against "Reappearing" conceit: on the proper use of criticism. Trans/Form/Ação, Marília, v. 37, n. 3, p. 45-56, Set./Dez., 2014.

\begin{abstract}
The author argues that Vico's critique of conceit does not refer only to nations and cultures, and to philosophical-theological theories focused on origins in ancient and mythical times and on the relationship between sacred and profane history. Vico's critique is also an ethical-philosophical device that invites humanity, now at the height of the civilizing process, to guard against any form of conceit by nations (in contemporary terms: ethnocentric pride), as well as any self-important manifestations by intellectuals (i.e., all forms of anthropocentric philosophy and theory).
\end{abstract}

KEYWORDS: Ethnocentrism. Anthropocentrism. Ethics. Interculturalism. Giambattista Vico.

\title{
BIBLIOGRAFIA
}

BATTISTINI, Andrea. Note alla Scienza nuova del 1744: Vico, 1744. v. 2. 
CACCIATORE, Giuseppe. Metaphysik, Poesie und Geschichte: über die Philosophie von Giambattista Vico. Berlin: Akademie Verlag, 2002.

. L'infinito nella storia: saggi su Vico. Napoli: Edizioni Scientifiche Italiane, 2009.

. Le "borie" di Vico tra etica e filosofia della storia. Rivista di Filosofia, Bologna, v. 102, n. 3, p. 363-380, 2011.

. Per una critica della ragione poetica: l'“altra” razionalità di Vico. In: VANZULLI, Marco (Ed.). Razionalità e modernità in Vico. Milano-Udine: Mimesis, 2012. p. 109-128.

CAPONIGRI, Robert, A. Filosofia e filologia: la "nuova arte della critica" di Giambattista Vico. Bollettino del Centro di Studi Vichiani, Napoli, v. 22/23, p. 29-61, 1982-1983.

CASSIRER, Ernst. Die Philosophie der Aufklärung. Tübingen: Mohr. 1932. Trad. it.: La filosofia dell'Illuminismo. Firenze: La Nuova Italia, 1970.

DIANA, Rosario. Andrea Sorrentino e la "boria" universalistica di Vico: un confronto fruttuoso. In: SORRENTINO, Andrea. Vico e la cultura mediterranea. Roma: Edizioni di Storia e Letteratura, 2011. p. 71-82.

DILTHEY, Wilhelm. Critica della ragione storica. Torino: Einaudi, 1954.

KOSELLECK, Reinhart. Kritik und Krise: ein Beitrag zur Pathogenese der bürgerlichen Welt. Freiburg: Alber, 1959. Trad. it.: Critica illuministica e crisi della società borghese. Bologna: il Mulino, 1972.

ORTEGA Y GASSET, José. Ni vitalismo ni razionalismo. In: Obras Completas. Madrid: Taurus, 2005. t. 3.

OTTO, Stephan. Giambattista Vico: lineamenti della sua filosofia. Napoli: Guida, 1992.

PACI, Enzo. Vico, lo strutturalismo e l'enciclopedia fenomenologica delle scienze. In: Idee per una enciclopedia fenomenologica. Milano: Bompiani, 1973.

PONS, Alain. Boria delle nazioni et boria de' dotti. Vico, le mythe de l'Égipte et les Hièroglyphes: la redécouverte de la Grèce et de l' Égipte au XVIII siècle. Nantes: Crini, 1997. (Actes du colloque 18-21 mai 1995).

VERENE, Donald, P. Vico's science of imagination. Ithaca: Cornell University Press, 1981. Trad. it.: Vico. La scienza della fantasia. Roma: Armando, 1984.

VICO, Giambattista. Scienza nuova 1725. In: . Opere. Milano: Mondadori, 1990. v. 2. Scienza nuova 1744: In: . Opere. Milano: Mondadori, 1990. v. 1. Epistole: con aggiunte le epistole dei suoi corrispondenti. Napoli: Morano, 1992.

Enviado em: 10/09/2014

Aceito em: 30/09/2014 
CACCIATORE, G. 